\title{
Bioremediation Potential of Native Hydrocarbons Degrading Bacteria in Crude Oil Polluted Soil
}

\author{
Mariana MARINESCU ${ }^{1}$, Anca LACATUSU ${ }^{1}$, Eugenia GAMENT ${ }^{1}$, Georgiana PLOPEANU ${ }^{1}$, Vera \\ CARABULEA $^{1}$
}

National Research and Development Institute for Soil Science, Agrochemistry and Environment, Romania

*Corresponding author: mara.marinescu@icpa.ro

Bulletin USAMV series Agriculture 74(1)/2017

Print ISSN 1843-5246; Electronic ISSN 1843-5386

DOI 10.15835/buasvmcn-agr: 12654

\begin{abstract}
Bioremediation of crude oil contaminated soil is an effective process to clean petroleum pollutants from the environment. Crude oil bioremediation of soils is limited by the bacteria activity in degrading the spills hydrocarbons. Native crude oil degrading bacteria were isolated from different crude oil polluted soils. The isolated bacteria belong to the genera Pseudomonas, Mycobacterium, Arthrobacter and Bacillus. A natural biodegradable product and bacterial inoculum were used for total petroleum hydrocarbon (TPH) removal from an artificial polluted soil. For soil polluted with $5 \%$ crude oil, the bacterial top, including those placed in the soil by inoculation was 30 days after impact, respectively 7 days after inoculum application, while in soil polluted with $10 \%$ crude oil, multiplication top of bacteria was observed in the determination made at 45 days after impact and 21 days after inoculum application, showing once again how necessary is for microorganisms habituation and adaptation to environment being a function of pollutant concentration. The microorganisms inoculated showed a slight adaptability in soil polluted with $5 \%$ crude oil, but complete inhibition in the first 30 days of experiment at $10 \%$ crude oil.
\end{abstract}

Keywords: bioremediation, crude oil, native hydrocarbons degrading bacteria, polluted soil

\section{INTRODUCTION}

Due to the persistence nature of crude oil, it remains in the environment for long time, which affects the soil quality by changing the physical, physiological, biochemical properties and microbial diversity (Margesin et al., 2013). Plants are also susceptible to the crude oil exposure, due to phytotoxic nature of crude oil and immobilization of availability of plant nutrient in soil (Udo, and Fayemi, 1995)]. Physical, chemical and thermal methods have commonly been employed to clean up the crude oil polluted soils (Roy et al., 2016). Techniques are relatively expensive and require site restoration (Lundstedt et al., 2003). Biological methods are relatively affordable and do not introduce any additional chemicals to the environment. These bacterial strains have potential in recovering the crude oil polluted soils as well as improving plant health (Glick, 2010). The rate of crude oil degradation using beneficial microorganisms had also been augmented in the field by supplying limiting nutrients to the affected sites (Atlas, 1991). Bioaugmentation involves inoculating exogenous degrading microorganisms to the soil (Taccari et al., 2012; Wu et al., 2016). Biostimulation stimulates the degrading capacity of the indigenous community by adding nutrients to avoid metabolic limitations (Abed et al., 2014). Currently, many reports have shown that bioaugmentation and biostimulation enhance the biodegradation of hydrocarbons in crude oil polluted soil (Suja et al., 2014). Because the process of bioremediation is carried out by various microorganisms in soil, understanding how bio- 
augmentation and biostimulation influence the populations of hydrocarbon degraders, the diversity and activity of the microbial community, and the ability of bacterial inoculum to adapt to new environmental conditions are very important in ensuring effective bioremediation of crude oil polluted soil (Liu et al., 2012).

\section{MATERIALS AND METHODS}

The main objective of this research is to test the capacity to increase the biodegradation of crude oil by stimulating the bacteria. To achieve data concerning the bioremediation of polluted soil with crude oil was realized a greenhouse experiment. The soil used for this experiment was a calcic chernozems. This type of soil was chosen because of its physical, chemical and biological properties favorable to plant growth. The study focused on the application of the two major technologies known in bioremediation method such as: soil biostimulation based on environmental conditions improvement for microorganisms multiplication and activity to degrade crude oil, and bioaugmentation based on enriching the soil with specific biodegrading hydrocarbons microorganisms. Biostimulation - the first technological link included an innovation element based on using an organic compound made from cellulose fibers for soil polluted conditioning with additives to optimize its structure, water and air circulation regime in soil, and not least achieving a protective interface between degrading microorganisms and pollutant.

Ecosol compound was chosen for experiment by analysing a series of organic compounds suitable for conditioning soil polluted with organic pollutants, especially because of its biodegradability properties. Bioaugmentation - the second technology link was achieved by soil inoculation with bacterial bioproducts made from specific bacteria selected and tested in the laboratory for their ability to degrade crude oil.

The experiment was set up by an artificial pollution of soil with different quantities of ECOSOL. After 21 days from pollution, the soil was inoculated with bacteria. The bacterial inoculum was developed from microorganisms that occur naturally in the soil like Pseudomonas, Mycobacterium, Arthrobacter globiformis and Bacillus megaterium.

The experimental variants are: $V_{1}$, control (unpolluted soil); $\mathrm{V}_{2}$, polluted soil with $5 \%$ crude oil; $\mathrm{V}_{3}$, polluted soil with $10 \%$ crude oil; $\mathrm{V}_{4}$, polluted soil with $5 \%$ crude oil $+0.25 \%$ Ecosol; $\mathrm{V}_{5}$, polluted soil with $5 \%$ crude oil $+0.25 \%$ Ecosol + bacterial inoculum; $\mathrm{V}_{6}$, polluted soil with $5 \%$ crude oil + $0.5 \%$ Ecosol; $V_{7}$, polluted soil with $5 \%$ crude oil $+0.5 \%$ Ecosol + bacterial inoculum; $V_{8}$, polluted soil with $10 \%$ crude oil $+0.5 \%$ Ecosol; $\mathrm{V}_{9}$, polluted soil with $10 \%$ crude oil $+0.25 \%$ Ecosol + bacterial inoculum; $\mathrm{V}_{10}$, polluted soil with $10 \%$ crude oil + $1 \%$ Ecosol; $V_{11}$, polluted soil with $10 \%$ crude oil + $1 \%$ Ecosol + bacterial inoculum.

\section{RESULTS AND DISCUSSIONS}

Bacterial inoculum application after 21 days from the experiment beginning was reflected in very significant increases in the levels of total heterotrophic bacteria determined in polluted soil. Immediately after artificial soil pollution with crude oil, at impact, bacterial populations have decreased the multiplication rate, and later, after an adaptation and selection process, the resistance component will extensively proliferate.

For soil polluted with $5 \%$ crude oil, the bacterial top, including those placed in the soil by inoculation was 30 days after impact, respectively 7 days after inoculum application, while in soil polluted with $10 \%$ crude oil, multiplication top of bacteria was observed in the determination made at 45 days after impact and 21 days after inoculum application, showing once again how necessary is for microorganisms habituation and adaptation to environment being a function of pollutant concentration. In soil excessively polluted with crude oil, bacterial population size in conditioned variant with Ecosol maximum dose (1\%) presented values comparable to those of inoculated variants, demonstrating the protective and stimulation effect of soil bacteria, including those involved in the degradation of crude oil exercised by Ecosol organic compound applied.

The quantitative evolution of bacterial communities from soil polluted with crude oil clearly demonstrated that the Ecosol application decreases the time needed for microorganisms involved in crude oil biodegradation to adapt at substrate and conditions of pollution.

Frequent bacterial isolated from experimental variants, in most determination stages were: Pseudomonas, Arthrobacter, Mycobacterium, bacterial of the most reputable in terms of species with high capacities in hydrocarbon biodegradation. 
Isolates of Pseudomonas, Arthrobacter and Mycobacterium led to the establishment of inoculum applied to the soil, and very high frequency showed a better survival rate and their adaptability to environmental conditions.

Bacterial isolated as Pseudomonas and Arthrobacter dominated the heterotrophic bacteria population in the majority of experimental variants, occasionally accompanied by species belonging to the genera: Micrococcus, Enterobacter and Flavobacterium. The results are presented in table 1 and 2.

Dynamics of microorganisms inoculated showed a slight adaptability in soil polluted with $5 \%$ crude oil, but complete inhibition in the first 30 days of experiment at $10 \%$ crude oil.

After the acclimatization period by 30 days, the excessive concentration of pollutants has been massive multiplication of bacteria in inoculated variants, especially in the conditioned variant with maximum dose of Ecosol. At 45 days, there was a real explosion in variants of bacteria inoculated with total heterotrophic bacteria values double, triple compared with uninoculated variants. At 60 days, bacterial populations have begun to reduce their size, as the value of soil polluted with $10 \%$ crude oil and untreated. This demonstrates that the application reduces the time needed to adapt to Ecosol substrate for microorganisms involved in petroleum hydrocarbons biodegradation.

Bacterial microcosms developed on the surface of nutrient agar media (Topping and CZPA) most abundant were Arthrobacter and Pseudomonas strains, which were derived from both the inoculum is applied, and its own microflora of the soil (Figs 1-4).

The value of the total heterotrophic bacteria in soil is a response to a large number of factors, including crude oil content, which causes new ecological conditions with a strong impact on microbial evolution. These conditions affect not only the size, but also the diversity of bacterial communities. The soil is mainly colonized by bacterial genera species able to use hydrocarbons from crude oil in its metabolism.

Tab. 1. Diversity of heterotrophic bacterial microflora in the soil polluted with $5 \%$ crude oil

\begin{tabular}{|c|c|c|c|c|c|c|c|}
\hline $\begin{array}{c}\text { Experimental } \\
\text { variants }\end{array}$ & 7 days & 14 days & 21 days & 30 days & 45 days & 60 days & 90 days \\
\hline $\mathrm{V}_{1}-\underset{\text { soil }}{\text { unpolluted }}$ & $\begin{array}{c}\text { Arthrobacter } \\
\text { globiformis, } \\
\text { Bacillus cereus, } \\
\text { Pseudomonas } \\
\text { sp., } \\
\text { Streptomyces } \\
\text { sp. }\end{array}$ & $\begin{array}{c}\text { Bacillus } \\
\text { megaterium, } \\
\text { B. cereus, } \\
\text { B. polymyxa, } \\
\text { Arthrobacter } \\
\text { globiformis, } \\
\text { Pseudomonas sp. }\end{array}$ & $\begin{array}{l}\text { Arthrobacter } \\
\text { globiformis, } \\
\text { Bacillus cereus, } \\
\text { B. megaterium, } \\
\text { B. circulans, } \\
\text { B. polymyxa. }\end{array}$ & $\begin{array}{c}\text { Arthrobacter } \\
\text { globiformis, } \\
\text { Bacillus circulans } \\
\text { Pseudomonas sp., } \\
\text { Bacillus } \\
\text { megaterium, } \\
\text { B. cereus, } \\
\text { Streptomyces sp. }\end{array}$ & $\begin{array}{l}\text { Bacillus circulans, } \\
\text { Arthrobacter } \\
\text { globiformis, } \\
\text { Bacillus } \\
\text { polymyxa, } \\
\text { B. megaterium, } \\
\text { B. cereus. }\end{array}$ & $\begin{array}{c}\text { Arthrobacter } \\
\text { globiformis, } \\
\text { Bacillus circulans, } \\
\text { B. polymyxa, } \\
\text { Pseudomonas sp. }\end{array}$ & $\begin{array}{c}\text { Bacillus } \\
\text { megaterium, } \\
\text { B. circulans, } \\
\text { B. polymyxa, } \\
\text { B. cereus, } \\
\text { Streptomyces sp. }\end{array}$ \\
\hline $\begin{array}{c}\mathrm{V}_{2}-\text { soil polluted } \\
\text { with } 5 \% \text { crude } \\
\text { oil }\end{array}$ & $\begin{array}{l}\text { Pseudomonas } \\
\text { sp., } \\
\text { Arthrobacter } \\
\text { globiformis, } \\
\text { Bacillus } \\
\text { sphaericus. }\end{array}$ & $\begin{array}{c}\text { Pseudomonas } \\
\text { sp., } \\
\text { Arthrobacter } \\
\text { globiformis, } \\
\text { Bacillus } \\
\text { circulans. }\end{array}$ & $\begin{array}{c}\text { Pseudomonas } \\
\text { sp., } \\
\text { Bacillus } \\
\text { circulans, } \\
\text { Arthrobacter } \\
\text { globiformis. }\end{array}$ & $\begin{array}{c}\text { Pseudomonas } \\
\text { sp., } \\
\text { Arthrobacter } \\
\text { globiformis, } \\
\text { Bacillus } \\
\text { circulans, } \\
\text { B. polymyxa, } \\
\text { Arthrobacter } \\
\text { simplex } \\
\end{array}$ & $\begin{array}{c}\text { Pseudomonas } \\
\text { sp., } \\
\text { Arthrobacter } \\
\text { globiformis, } \\
\text { Bacillus } \\
\text { polymyxa, } \\
\text { B. circulans, } \\
\text { Arthrobacter } \\
\text { simplex. } \\
\end{array}$ & $\begin{array}{l}\text { Pseudomonas sp., } \\
\text { Arthrobacter } \\
\text { globiformis, } \\
\text { Mycobacterium } \\
\text { roseum, } \\
\text { Bacillus circulans, } \\
\text { B. megaterium. }\end{array}$ & $\begin{array}{c}\text { Arthrobacter } \\
\text { globiformis, } \\
\text { Bacillus circulans, } \\
\text { B. polymyxa, } \\
\text { Pseudomonas sp., } \\
\text { Mycobacterium } \\
\text { phley, } \\
\text { Streptomyces sp. }\end{array}$ \\
\hline $\begin{array}{c}\mathrm{V}_{4}-\text { soil polluted } \\
\text { with } 5 \% \text { crude } \\
\text { oil }+0.25 \% \\
\text { Ecosol }\end{array}$ & $\begin{array}{l}\text { Arthrobacter } \\
\text { citreus, } \\
\text { Streptomyces } \\
\text { sp. }\end{array}$ & $\begin{array}{c}\text { Pseudomonas sp., } \\
\text { Arthrobacter } \\
\text { globiformis, } \\
\text { Bacillus } \\
\text { polymyxa. }\end{array}$ & $\begin{array}{l}\text { Pseudomonas sp., } \\
\text { Arthrobacter } \\
\text { globiformis, } \\
\text { Bacillus circulans. }\end{array}$ & $\begin{array}{c}\text { Bacillus circulans, } \\
\text { Arthrobacter } \\
\text { globiformis. }\end{array}$ & $\begin{array}{l}\text { Pseudomonas sp., } \\
\text { Bacillus circulans, } \\
\text { Arthrobacter } \\
\text { globiformis, } \\
\text { Bacillus } \\
\text { megaterium, } \\
\text { B. polymyxa, } \\
\text { B. sphaericus, } \\
\text { Arhtrobacter } \\
\text { citreus. }\end{array}$ & $\begin{array}{l}\text { Pseudomonas sp., } \\
\text { Arthrobacter } \\
\text { globiformis, } \\
\text { Bacillus polymyxa, } \\
\text { B. circulans, } \\
\text { Mycobacterium sp., } \\
\text { Bacillus cereus, } \\
\text { B. megaterium,, } \\
\text { B. macerans, } \\
\text { Arhtrobacter } \\
\text { simplex, } \\
\text { Bacillus sphaericus. }\end{array}$ & $\begin{array}{l}\text { Pseudomonas } \\
\text { sp., } \\
\text { Arthrobacter } \\
\text { simplex, } \\
\text { Bacillus } \\
\text { circulans, } \\
\text { B. megaterium, } \\
\text { Mycobacterium } \\
\text { phley, } \\
\text { Streptomyces sp. }\end{array}$ \\
\hline
\end{tabular}


Tab. 1. Diversity of heterotrophic bacterial microflora in the soil polluted with $5 \%$ crude oil - continued

\begin{tabular}{|c|c|c|c|c|c|c|c|}
\hline $\begin{array}{c}\text { Experimental } \\
\text { variants }\end{array}$ & 7 days & 14 days & 21 days & 30 days & 45 days & 60 days & 90 days \\
\hline $\begin{array}{c}\mathrm{V}_{5}-\text { soil } \\
\text { polluted with } \\
5 \% \text { crude oil }+ \\
0.25 \% \text { Ecosol } \\
+ \text { bacterial } \\
\text { inoculum }\end{array}$ & $\begin{array}{l}\text { Pseudomonas } \\
\text { sp., } \\
\text { Arthrobacter } \\
\text { globiformis, } \\
\text { Bacillus } \\
\text { megaterium. }\end{array}$ & $\begin{array}{c}\text { Arthrobacter } \\
\text { globiformis, } \\
\text { Pseudomonas } \\
\text { sp., } \\
\text { Bacillus } \\
\text { circulans, } \\
\text { B. megaterium. }\end{array}$ & $\begin{array}{l}\text { Pseudomonas } \\
\text { sp., } \\
\text { Arthrobacter } \\
\text { globiformis, } \\
\text { Bacillus } \\
\text { polymyxa, } \\
\text { B. circulans. }\end{array}$ & $\begin{array}{c}\text { Pseudomonas } \\
\text { sp., } \\
\text { Arthrobacter } \\
\text { globiformis, } \\
\text { Bacillus } \\
\text { circulans, } \\
\text { B. megaterium, } \\
\text { Micrococcus. }\end{array}$ & $\begin{array}{c}\text { Pseudomonas sp., } \\
\text { Bacillus circulans, } \\
\text { Arthrobacter } \\
\text { globiformis, } \\
\text { Mycobacterium } \\
\text { roseum, } \\
\text { Bacillus polymyxa, } \\
\text { B. megaterium, } \\
\text { B. sphaericus, } \\
\text { Enterobacter. }\end{array}$ & $\begin{array}{l}\text { Pseudomonas sp., } \\
\text { Arthrobacter simplex, } \\
\text { Bacillus circulans, } \\
\text { Mycobacterium sp., } \\
\text { Bacillus sphaericus, } \\
\text { B. megaterium, } \\
\text { Arthrobacter } \\
\text { globiformis. }\end{array}$ & $\begin{array}{l}\text { Pseudomonas sp., } \\
\text { Arthrobacter } \\
\text { globiformis, } \\
\text { Mycobacterium } \\
\text { roseum, } \\
\text { Bacillus circulans, } \\
\text { Streptomyces sp. }\end{array}$ \\
\hline $\begin{array}{l}\mathrm{V}_{6}-\text { soil polluted } \\
\text { with } 5 \% \text { crude } \\
\text { oil }+0.5 \% \text { Ecosol }\end{array}$ & $\begin{array}{l}\text { Pseudomonas } \\
\text { sp., } \\
\text { Bacillus cereus, } \\
\text { Arthrobacter } \\
\text { globiformis, } \\
\text { Bacillus } \\
\text { megaterium. }\end{array}$ & $\begin{array}{l}\text { Pseudomonas } \\
\text { sp., } \\
\text { Bacillus cereus, } \\
\text { Arthrobacter } \\
\text { globiformis, } \\
\text { Bacillus } \\
\text { megaterium, } \\
\text { B. polymyxa. }\end{array}$ & $\begin{array}{c}\text { Pseudomonas } \\
\text { sp., } \\
\text { Bacillus cereus, } \\
\text { Arthrobacter } \\
\text { globiformis, } \\
\text { Bacillus } \\
\text { megaterium } \\
\text { Bacillus } \\
\text { circulans. }\end{array}$ & $\begin{array}{c}\text { Pseudomonas } \\
\text { sp., } \\
\text { Bacillus } \\
\text { circulans, } \\
\text { B. polymyxa, } \\
\text { B. megaterium. }\end{array}$ & $\begin{array}{l}\text { Pseudomonas sp., } \\
\text { Mycobacterium phley, } \\
\text { Arthrobacter simplex, } \\
\text { Bacillus polymyxa, } \\
\text { B. circulans, } \\
\text { B. sphaericus, } \\
\text { B. megaterium. }\end{array}$ & $\begin{array}{l}\text { Pseudomonas sp., } \\
\text { Bacillus circulans, } \\
\text { Arthrobacter simplex, } \\
\text { Bacillus polymyxa. }\end{array}$ & $\begin{array}{l}\text { Bacillus cereus } \\
\text { var. mycoides, } \\
\text { Pseudomonas sp., } \\
\text { Bacillus circulans, } \\
\text { Arthrobacter } \\
\text { globiformis, } \\
\text { Mycobacterium } \\
\text { roseum. }\end{array}$ \\
\hline $\begin{array}{l}\text { V7 - soil polluted } \\
\text { with } 5 \% \text { crude } \\
\text { oil + } 0.5 \% \text { Ecosol } \\
\quad+\text { bacterial } \\
\text { inoculum }\end{array}$ & $\begin{array}{l}\text { Pseudomonas } \\
\text { sp., } \\
\text { Arthrobacter } \\
\text { citreus, } \\
\text { Streptomyces } \\
\text { sp. }\end{array}$ & $\begin{array}{c}\text { Pseudomonas } \\
\text { sp., } \\
\text { Arthrobacter } \\
\text { globiformis, } \\
\text { Bacillus } \\
\text { circulans, } \\
\text { Streptomyces } \\
\text { sp. }\end{array}$ & $\begin{array}{l}\text { Pseudomonas } \\
\text { sp., } \\
\text { Arthrobacter } \\
\text { globiformis, } \\
\text { Bacillus } \\
\text { polymyxa. }\end{array}$ & $\begin{array}{c}\text { Pseudomonas } \\
\text { sp., } \\
\text { Arthrobacter } \\
\text { globiformis, } \\
\text { Bacillus } \\
\text { circulans } \\
\text { Mycobacterium } \\
\text { phley, Bacillus } \\
\text { polymyxa. }\end{array}$ & $\begin{array}{l}\text { Pseudomonas sp., } \\
\text { Arthrobacter } \\
\text { globiformis, } \\
\text { Bacillus polymyxa, } \\
\text { B. circulans, } \\
\text { Mycobacterium } \\
\text { roseum, } \\
\text { Bacillus megaterium, } \\
\text { B. sphaericus, } \\
\text { B. macerans. }\end{array}$ & $\begin{array}{c}\text { Pseudomonas sp., } \\
\text { Arthrobacter } \\
\text { globiformis, } \\
\text { Bacillus polymyxa, } \\
\text { B. circulans, } \\
\text { B. sphaericus, } \\
\text { B. megaterium, } \\
\text { Arthrobacter simplex, } \\
\text { Mycobacterium phley, } \\
\text { Flovobacterium. }\end{array}$ & $\begin{array}{c}\text { Arthrobacter } \\
\text { globiformis, } \\
\text { Pseudomonas } \\
\text { sp., } \\
\text { Bacillus } \\
\text { circulans, } \\
\text { B. macerans. }\end{array}$ \\
\hline
\end{tabular}
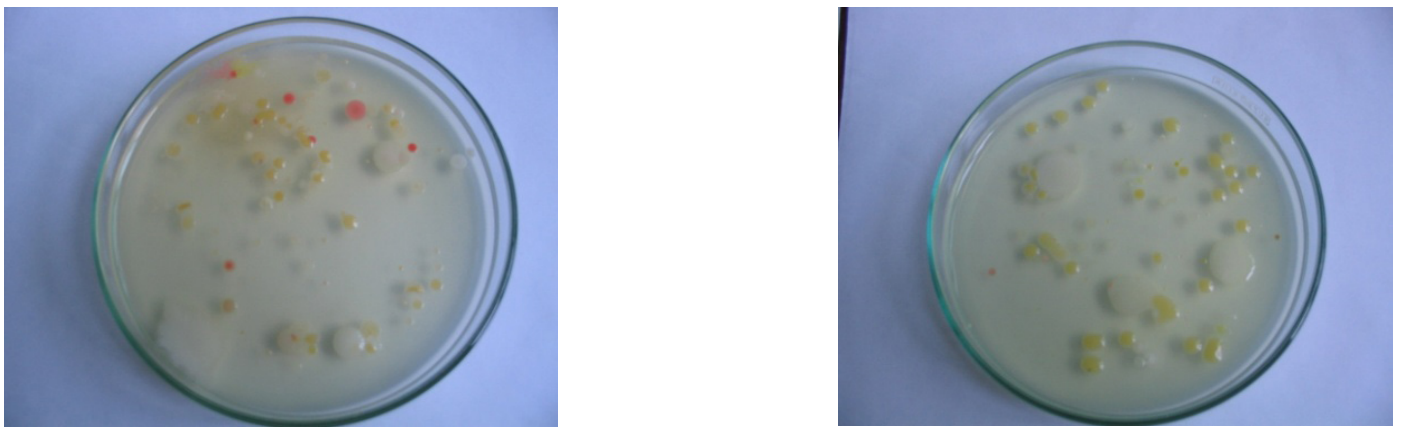

Fig. 1. Bacterial microcosm (Topping medium)

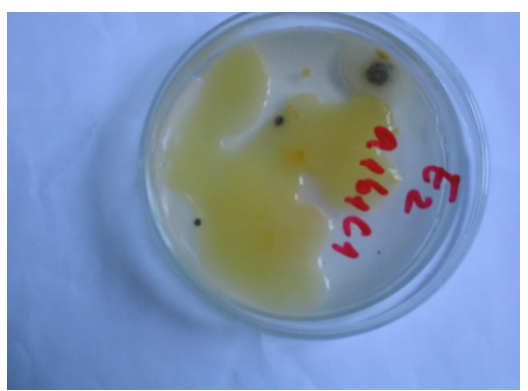

Fig. 2. Macroscopic appearance - Pseudomonas sp. colonies on Topping medium (experimental variant V8) 
Tab 2. Diversity of heterotrophic bacterial microflora in the soil polluted with $10 \%$ crude oil

\begin{tabular}{|c|c|c|c|c|c|c|c|}
\hline $\begin{array}{c}\text { Experimental } \\
\text { variants }\end{array}$ & 7 days & 14 days & 21 days & 30 days & 45 days & 60 days & 90 days \\
\hline $\mathrm{V}_{1}-\underset{\text { soil }}{\text { unpolluted }}$ & $\begin{array}{c}\text { Arthrobacter } \\
\text { globiformis, } \\
\text { Bacillus cereus, } \\
\text { Pseudomonas } \\
\text { sp., } \\
\text { Streptomyces } \\
\text { sp. }\end{array}$ & $\begin{array}{c}\text { Bacillus } \\
\text { megaterium, } \\
\text { B. cereus, } \\
\text { B. polymyxa, } \\
\text { Arthrobacter } \\
\text { globiformis, } \\
\text { Pseudomonas } \\
\text { sp. }\end{array}$ & $\begin{array}{l}\text { Arthrobacter } \\
\text { globiformis, } \\
\text { Bacillus cereus, } \\
\text { B. megaterium, } \\
\text { B. circulans, } \\
\text { B. polymyxa. }\end{array}$ & $\begin{array}{l}\text { Arthrobacter } \\
\text { globiformis, } \\
\text { Bacillus } \\
\text { circulans } \\
\text { Pseudomonas } \\
\text { sp., } \\
\text { Bacillus } \\
\text { megaterium, } \\
\text { B. cereus, } \\
\text { Streptomyces sp. }\end{array}$ & $\begin{array}{l}\text { Bacillus circulans, } \\
\text { Arthrobacter } \\
\text { globiformis, } \\
\text { Bacillus polymyxa, } \\
\text { B. megaterium, } \\
\text { B. cereus. } \\
\end{array}$ & $\begin{array}{l}\text { Arthrobacter } \\
\text { globiformis, } \\
\text { Bacillus circulans, } \\
\text { B. polymyxa, } \\
\text { Pseudomonas sp. }\end{array}$ & $\begin{array}{c}\text { Bacillus } \\
\text { megaterium, } \\
\text { B. circulans, } \\
\text { B. polymyxa, } \\
\text { B. cereus, } \\
\text { Streptomyces sp. }\end{array}$ \\
\hline $\begin{array}{l}\text { V3 - soil } \\
\text { polluted with } \\
\text { 10\% crude oil }\end{array}$ & $\begin{array}{c}\text { Pseudomonas } \\
\text { sp., } \\
\text { Arthrobacter } \\
\text { globiformis, } \\
\text { Streptomyces } \\
\text { sp. }\end{array}$ & $\begin{array}{l}\text { Pseudomonas } \\
\text { sp., } \\
\text { Arthrobacter } \\
\text { globiformis, } \\
\text { Bacillus } \\
\text { megaterium. }\end{array}$ & $\begin{array}{l}\text { Arthrobacter } \\
\text { globiformis, } \\
\text { Pseudomonas } \\
\text { sp., } \\
\text { Bacillus } \\
\text { megaterium. }\end{array}$ & $\begin{array}{c}\text { Pseudomonas } \\
\text { sp., Arthrobacter } \\
\text { globiformis, } \\
\text { Bacillus } \\
\text { circulans, } \\
\text { Streptomyces sp. }\end{array}$ & $\begin{array}{c}\text { Pseudomonas sp., } \\
\text { Arthrobacter } \\
\text { globiformis, } \\
\text { Mycobacterium sp., } \\
\text { Bacillus } \\
\text { megaterium, } \\
\text { B. circulans, } \\
\text { Arthrobacter } \\
\text { simplex, } \\
\text { Bacillus sphaericus. }\end{array}$ & $\begin{array}{c}\text { Pseudomonas sp., } \\
\text { Arthrobacter simplex, } \\
\text { Bacillus circulans, } \\
\text { B. polymyxa, } \\
\text { Arthrobacter } \\
\text { globiformis, } \\
\text { Bacillus megaterium, } \\
\text { B. cereus, } \\
\text { B. macerans. } \\
\end{array}$ & $\begin{array}{c}\text { Pseudomonas sp., } \\
\text { Arthrobacter } \\
\text { citreus, } \\
\text { Bacillus circulans, } \\
\text { Enterobacter, } \\
\text { Streptomyces sp. }\end{array}$ \\
\hline $\begin{array}{c}\mathrm{V}_{8}-\text { soil } \\
\text { polluted with } \\
10 \% \text { crude oil + } \\
0.5 \% \text { Ecosol }\end{array}$ & $\begin{array}{l}\text { Arthrobacter } \\
\text { globiformis, } \\
\text { Pseudomonas } \\
\text { sp., } \\
\text { Streptomyces } \\
\text { sp. }\end{array}$ & $\begin{array}{l}\text { Arthrobacter } \\
\text { globiformis, } \\
\text { Bacillus } \\
\text { circulans, } \\
\text { B. cereus, } \\
\text { Mycobacterium } \\
\text { sp. }\end{array}$ & $\begin{array}{l}\text { Pseudomonas } \\
\text { sp., } \\
\text { Arthrobacter } \\
\text { globiformis, } \\
\text { Bacillus } \\
\text { megaterium, } \\
\text { Streptomyces } \\
\text { sp. }\end{array}$ & $\begin{array}{c}\text { Bacillus } \\
\text { circulans, } \\
\text { Mycobacterium } \\
\text { sp., } \\
\text { Bacillus } \\
\text { megaterium. }\end{array}$ & $\begin{array}{c}\text { Bacillus cereus var. } \\
\text { mycoides, } \\
\text { Pseudomonas sp., } \\
\text { Arthrobacter } \\
\text { globiformis, } \\
\text { Mycobacterium } \\
\text { phley, } \\
\text { Bacillus } \\
\text { megaterium. }\end{array}$ & $\begin{array}{c}\text { Pseudomonas sp., } \\
\text { Bacillus circulans, } \\
\text { Arthrobacter } \\
\text { globiformis, } \\
\text { Bacillus polymyxa, } \\
\text { Mycobacterium } \\
\text { roseum, } \\
\text { Bacillus megaterium. }\end{array}$ & $\begin{array}{c}\text { Arthrobacter } \\
\text { globiformis, } \\
\text { Pseudomonas sp., } \\
\text { Bacillus } \\
\text { polymyxa, } \\
\text { Bacillus circulans. }\end{array}$ \\
\hline $\begin{array}{c}\mathrm{V}_{9}-\text { soil } \\
\text { polluted with } \\
10 \% \text { crude oil } \\
+0.5 \% \text { Ecosol } \\
+ \text { bacterial } \\
\text { inoculum }\end{array}$ & $\begin{array}{l}\text { Pseudomonas } \\
\text { sp., } \\
\text { Mycobacterium } \\
\text { roseum. }\end{array}$ & $\begin{array}{c}\text { Pseudomonas } \\
\text { sp., } \\
\text { Mycobacterium } \\
\text { sp., } \\
\text { Bacillus } \\
\text { circulans. }\end{array}$ & $\begin{array}{c}\text { Mycobacterium } \\
\text { phley, } \\
\text { Pseudomonas } \\
\text { sp., } \\
\text { Bacillus } \\
\text { circulans. }\end{array}$ & $\begin{array}{l}\text { Pseudomonas } \\
\text { sp., Arthrobacter } \\
\text { simplex, Bacillus } \\
\text { circulans, } \\
\text { B. polymyxa. }\end{array}$ & $\begin{array}{c}\text { Pseudomonas sp., } \\
\text { Bacilus circulans, } \\
\text { Micrococcus, } \\
\text { Bacillus polymyxa, } \\
\text { Arthrobacter } \\
\text { globiformis, } \\
\text { Mycobacterium } \\
\text { phley, } \\
\text { Bacillus } \\
\text { megaterium, } \\
\text { Arthrobacter } \\
\text { citreus. }\end{array}$ & $\begin{array}{l}\text { Pseudomonas sp., } \\
\text { Arthrobacter simplex, } \\
\text { Bacilus polymyxa, } \\
\text { B. circulans, } \\
\text { Arthrobacter citreus, } \\
\text { Mycobacterium phley, } \\
\text { Bacillus megaterium, } \\
\text { B. sphaericus, } \\
\text { B. cereus. }\end{array}$ & $\begin{array}{c}\text { Pseudomonas sp., } \\
\text { Mycobacterium } \\
\text { phley, } \\
\text { Arthrobacter } \\
\text { globiformis, } \\
\text { Bacilus circulans, } \\
\text { Arthrobacter } \\
\text { citreus, } \\
\text { Bacillus } \\
\text { megaterium. }\end{array}$ \\
\hline $\begin{array}{l}\mathrm{V}_{10}-\text { soil } \\
\text { polluted with } \\
10 \% \text { crude oil } \\
+1 \% \text { Ecosol }\end{array}$ & $\begin{array}{l}\text { Pseudomonas } \\
\text { sp., } \\
\text { Arthrobacter } \\
\text { globiformis. }\end{array}$ & $\begin{array}{l}\text { Pseudomonas } \\
\text { sp., } \\
\text { Arthrobacter } \\
\text { globiformis. }\end{array}$ & $\begin{array}{c}\text { Pseudomonas } \\
\text { sp., } \\
\text { Arthrobacter } \\
\text { globiformis, } \\
\text { Mycobacterium } \\
\text { sp. }\end{array}$ & $\begin{array}{c}\text { Pseudomonas sp., } \\
\text { Arthrobacter } \\
\text { globiformis, } \\
\text { Bacillus } \\
\text { circulans, } \\
\text { B. polymyxa. }\end{array}$ & $\begin{array}{c}\text { Pseudomonas sp., } \\
\text { Arthrobacter } \\
\text { globiformis, } \\
\text { Bacillus sphaericus, } \\
\text { B. circulans, } \\
\text { Mycobacterium sp., } \\
\text { Bacillus } \\
\text { megaterium, } \\
\text { Arthrobacter } \\
\text { simplex, } \\
\text { Bacillus polymyxa. }\end{array}$ & $\begin{array}{c}\text { Pseudomonas sp., } \\
\text { Arthrobacter citreus, } \\
\text { Bacillus circulans, } \\
\text { B. polymyxa, } \\
\text { Arthrobacter } \\
\text { globiformis, } \\
\text { Bacillus megaterium, } \\
\text { Mycobacterium } \\
\text { roseum, } \\
\text { Bacillus sphaericus, } \\
\text { Arthrobacter simplex. }\end{array}$ & $\begin{array}{l} \\
\text { Pseudomonas sp., } \\
\text { Arthrobacter } \\
\text { globiformis, } \\
\text { Mycobacterium } \\
\text { roseum, } \\
\text { Bacillus circulans. }\end{array}$ \\
\hline $\begin{array}{l}\mathrm{V}_{11}-\text { soil } \\
\text { polluted with } \\
10 \% \text { crude oil } \\
+1 \% \text { Ecosol } \\
+ \text { bacterial } \\
\text { inoculum }\end{array}$ & $\begin{array}{c}\text { Pseudomonas } \\
\text { sp., } \\
\text { Mycobacterium } \\
\text { phley. }\end{array}$ & $\begin{array}{c}\text { Pseudomonas } \\
\text { sp., } \\
\text { Mycobacterium } \\
\text { sp., } \\
\text { Arthrobacter } \\
\text { globiformis. }\end{array}$ & $\begin{array}{c}\text { Pseudomonas } \\
\text { sp., } \\
\text { Mycobacterium } \\
\text { roseum, } \\
\text { Arthrobacter } \\
\text { globiformis. }\end{array}$ & $\begin{array}{c}\text { Pseudomonas sp., } \\
\text { Arthrobacter } \\
\text { globiformis, } \\
\text { Mycobacterium } \\
\text { sp., } \\
\text { Bacillus } \\
\text { megaterium, } \\
\text { B. circulans. }\end{array}$ & $\begin{array}{c}\text { Pseudomonas sp., } \\
\text { Mycobacterium } \\
\text { phley, } \\
\text { Bacillus circulans, } \\
\text { Arthrobacter } \\
\text { globiformis, } \\
\text { Bacillus } \\
\text { megaterium, } \\
\text { B. polymyxa, } \\
\text { Arthrobacter } \\
\text { citreus, } \\
\text { Bacillus macerans. }\end{array}$ & $\begin{array}{l}\text { Pseudomonas sp., } \\
\text { Mycobacterium phley, } \\
\text { Bacillus circulans, } \\
\text { Arthrobacter } \\
\text { globiformis, } \\
\text { Bacillus polymyxa, } \\
\text { Flavobacterium, } \\
\text { Bacillus sphaericus, } \\
\text { B. megaterium. }\end{array}$ & $\begin{array}{l} \\
\text { Pseudomonas sp., } \\
\text { Arthrobacter } \\
\text { globiformis, } \\
\text { Bacillus circulans. }\end{array}$ \\
\hline
\end{tabular}



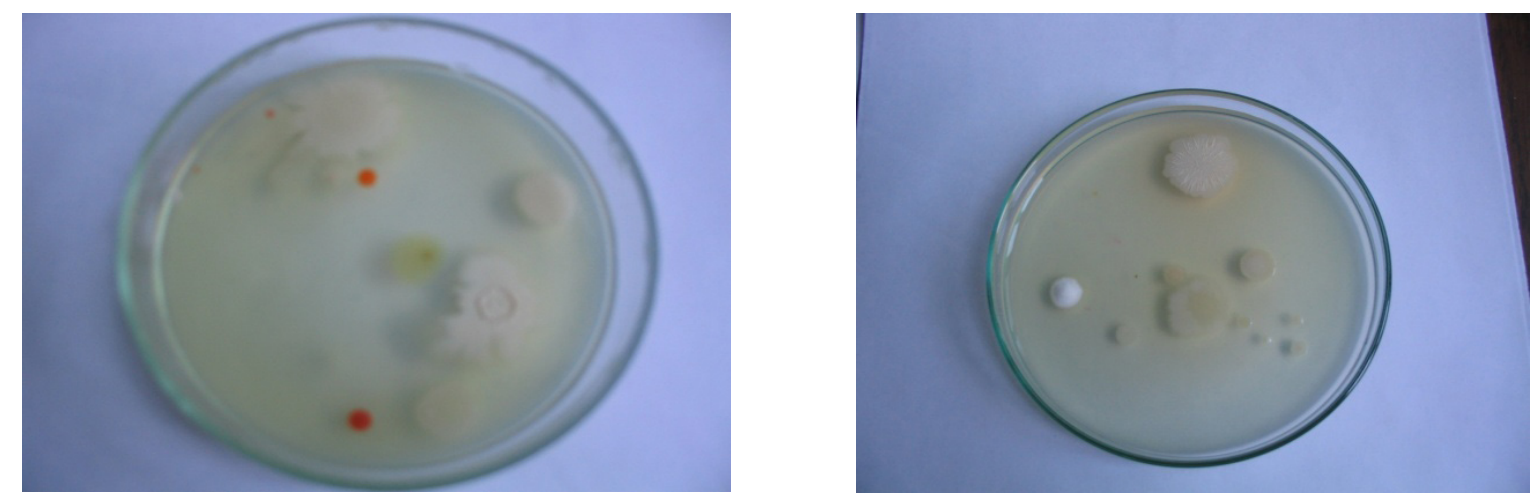

Fig. 3. Bacterial microcosm from control variant

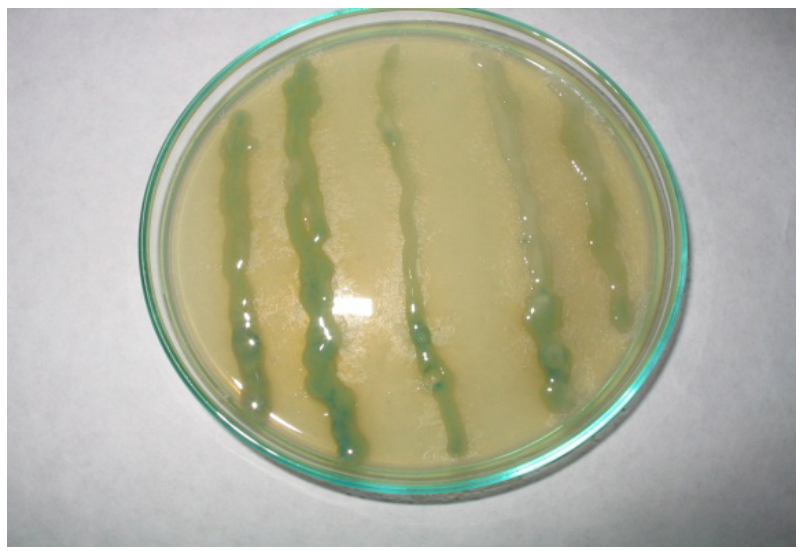

Fig. 4. Pseudomonas sp. - growth on CZPA medium

Survival of microorganisms in crude oil in uninoculated variants and after bacterial inoculation in the inoculated variants is a key deciding factor in the rate of biodegradation of hydrocarbons in soils.

\section{CONCLUSION}

The study focused on the application of the two major technologies known in bioremediation method such as: soil biostimulation based on environmental conditions improvement for microorganisms multiplication and activity to degrade crude oil, and bioaugmentation based on enriching the soil with specific biodegrading hydrocarbons microorganisms. For soil polluted with $5 \%$ crude oil, the bacterial top, including those placed in the soil by inoculation was 30 days after impact, respectively 7 days after inoculum application, while in soil polluted with $10 \%$ crude oil, multiplication top of bacteria was observed in the determination made at 45 days after impact and 21 days after inoculum application, showing once again how necessary is for microorganisms habituation and adaptation to environment being a function of pollutant concentration. In the most determination periods were determined Pseudomonas, Arthrobacter, Mycobacterium as frequent bacterial isolated from experimental variants. Isolates of Pseudomonas, Arthrobacter and Mycobacterium led to the establishment of inoculum applied to the soil, and very high frequency showed a better survival rate and their adaptability to environmental conditions. Bacterial isolated as Pseudomonas and Arthrobacter dominated the heterotrophic bacteria population in the majority of experimental variants, occasionally accompanied by species belonging to the genera: Micrococcus, Enterobacter and Flavobacterium.

Acknowledgements. The financial support provided by the UEFISCDI - Executive Unit for FinancingEducation Higher Research Development 
and Innovative - project no. 91/2014 - BIORESOL and PN 16.07.03.03/2016.

\section{REFERENCES}

1. Abed RMM, Al-Sabahi J, Al-Maqrashi F, Al-Habsi A, Al-Hinai M (2014). Characterization of hydrocarbon-degrading bacteria isolated from oil-contaminated sediments in the Sultanate of Oman and evaluation of bioaugmentation and biostimulation approaches in microcosm experiments. Int. Biodeterior. Biodegr. 89: 58-66,.

2. Atlas RM (1981). Microbial degradation of petroleum hydrocarbons: an environmental perspective. Microbiol. Rev. 45:180-209.

3. Glick BR (2010).Using soil bacteria to facilitate phytoremediation. Biotechnol. Adv. 28:367-374.

4. Liu PWG, Wang SY, Huang SG, Wang MZ, (2012). Effects of soil organic matter and ageing on remediation of diesel oil-contaminated soil and on the microbial ecology. Environ. Technol. 33: 2661-2672.

5. Lundstedt S, Haglund P, Oberg L (2003). Degradation and formation of polycyclic aromatic compounds during bioslurry treatment of an aged gasworks soil. Environ. Toxicol. Chem. 22:1413-1420.

6. Margesin, R., Labbe, D., Schinner, F., Greer, C., Whyte, L., (2003). Characterization of hydrocarbon-degrading microbial populations in contaminated and pristine alpine soils. Appl. Environ. Microbiol. 69, pp. 3085-3092.

7. Roy AS, Baruah R, Borah M, Singh AK, Boruah HP, Saikia N, Deka M, Dutta N, Bora TC (2016). Bioremediation potential of native hydrocarbon degrading bacterial strains in crude oil contaminated soil under microcosm study. International Biodeterioration \& Biodegradation 94:79-89.

8. Suja F, Rahim F, Taha MR, Hambali N, Razali MR, Khalid A., Hamzah A (2014). Effects of local microbial bioaugmentation and biostimulation on the bioremediation of total petroleum hydrocarbons (TPH) in crude oil contaminated soil based on laboratory and field observations. Int. Biodeterior. Biodegr. 90:115-122.

9. Taccari M, Milanovic V, Comitini F, Casucci C, Ciani M (2012). Effects of biostimulation and bioaugmentation on diesel removal and bacterial community. Int. Biodeterior. Biodegr. 66:39-46.

10. Udo EJ, Fayemi AAA (1995). The effect of oil pollution on soil germination, growth and nutrient uptake of corn. J. Environ. Qual. 4:537-540.

11. Wu, M., Dick, W.A., Li, W., Wang, X., Yang, Q., Wang, T., $\mathrm{Xu}$, L., Zhang, M., Chen L (2016). Bioaugmentation and biostimulation of hydrocarbon degradation and the microbial community in a petroleum-contaminated soil. International iodeterioration \& Biodegradation 107:158164. 